\title{
Learning of inverted and normally oriented faces in children and adults
}

ALVIN G. GOLDSTEIN

UNIVERSITY OF MISSOURI, COLUMBIA

\begin{abstract}
In a series of experiments, adult Ss learned paired associates composed of normally oriented faces and common proper names significantly faster than inverted faces and the same names. It was hypothesized that this difficulty with inverted faces was a function of the large number of exposures to normally oriented faces. To test this hypothesis young children (who, it was assumed, would have had far less experience with faces) and adults were asked to learn face-letter associations. Approximately half of each age group were shown upright face-letter associates; the remaining Ss were shown inverted face-letter associates. It was predicted that the difference in learning between the upright and inverted children's groups would be significantly smaller than the difference between the upright and inverted adult groups. This prediction was confirmed.
\end{abstract}

\section{Problem}

In a recent experiment, adult Ss were presented with a paired-associates learning task in which one member of the pair was a common proper name (e.g., John) or a capital letter, and the second member was a human face. The orientation in which faces were presented to Ss was varied; one group of Ss learned upright (Up) faces, while the other learned inverted (Inv) faces. We have found repeatedly that Up faces are learned significantly faster than Inv faces (Goldstein, 1962). In an attempt to understand this finding, it was hypothesized that for adult Ss, extreme overlearning of Up faces has occurred because of the high frequency of exposure to this stimulus. This overlearning interferes with learning an upside-down face. Because we can reasonably assume that young children have had fewer exposures to Up faces than adults, we predicted that children would learn Up and Inv facename associations with almost equal facility, while adults would learn the Up faces faster than the Inv. In other words, the performance of two groups of children learning Up and Inv faces will be more alike than the performance of two groups of adults learning similar stimuli.

Method

Each paired associate was a capital letter (C, F, H, $\mathrm{K}, \mathrm{L}, \mathrm{M}, \mathrm{S}, \mathrm{X})$ and a cropped, black-white photograph of a $13 \mathrm{yr}$. old face. In either the upright or inverted position, the identifying letter was always in an upright orientation below the face. Using the anticipation method, on the first trial, $S$ was shown each of the faceletter combinations for $5 \mathrm{sec}$. On all subsequent trials,
$\mathrm{S}$ was presented the face and had to report the letier. If $\mathrm{S}$ made no response to a face in $5 \mathrm{sec}$., the associated letter was uncovered. If $\mathrm{S}$ responded before the $5 \mathrm{sec}$. elapsed, the correct letter was also uncovered and S immediately knew whether his response was correct. A session was terminated either after the fifteenth trial, or after $\mathrm{S}$ attained the criterion of two consecutive errorless trials. Eight paired associates were thus presented to the adults, whereas the children were required to learn only four such pairs. Approximately half of the children were tested on four of the eight faces and the remaining faces were presented to the remaining children. Within the adult and children's groups, half learned the faces in an Up orientation and half learned them in an Inv orientation. However, only four of the eight letters were used with all the children ( $\mathrm{C}, \mathrm{H}, \mathrm{K}, \mathrm{X})$. Fifty-five second and third grade children ( 29 girls, 26 boys), and 48 college freshmen (24 women, $24 \mathrm{men}$ ) were individually tested in the experiment.

\section{Results}

To compare the adults' and children's data, per cent scores were calculated for each group from the total number correct on each of the 15 trials (Fig. 1). For each trial, a difference-score was calculated by subtracting the mean per cent correct responses to Inv faces from the mean per cent correct responses to Up faces. Separate difference-scores were calculated for children and for adults. The present experiment was designed to test the prediction that the children's

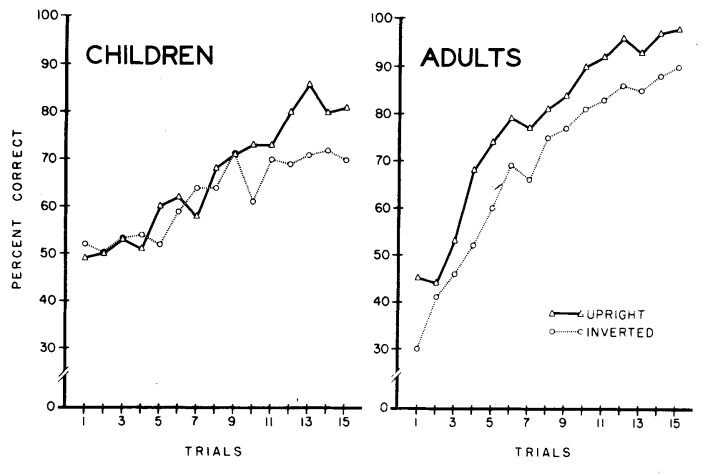

Fig. 1. Per cent correct responses to four paired associates for the children and eight paired associates for the adults. For the children, each data point in the Upright curve is derived from 24 Ss; in the Inverted curve, 31 Ss. All data points in the adult curves were derived from $24 \mathrm{Ss}$. 
difference-score would be significantly smaller than the adults' difference-score.

Figure 1 shows that among adults, per cent correct of the Inv Ss is below the per cent correct of the Up Ss at each of the 15 trials. In contrast, the children's curves overlap until trial 9, after which the performance of the Inv Ss falls below the Up Ss. The difference between the children's and adults' difference-scores was significant $(t=3.57 ; p<.01)$ in the predicted direction.

The results of analyses by sex are presented in Fig. 2 for boys and girls only; a similar analysis of the men's and women's data yielded curves almost identical to the Adult curves in Fig. 1, and therefore has not been included here. The boys' differencescore was statistically compared to the men's difference-score; this computation was also performed on the girls' and women's data. In both analyses, the adults' difference-scores were significantly larger than the children's scores $(t=2.11 ; p<.05$ for the girls vs women and $t=3.08 ; p<.01$ for the boys vs men). The analysis also suggests that inverting the stimulus significantly reduced the girls' performance $(t=2.30$; $\mathrm{p}<.05)$ but did not reduce the boys' performance $(t=1.09 ; p>.05)$.

\section{Diseussion}

As predicted, the childrens' Up-Inv difference-score was significantly smaller than the adults' Up-Inv difference-score. This finding supports the hypothesis that, by increasing the number of exposures to upright faces it becomes more difficult to make use of aspects of inverted faces during the learning task. In more general terms, a stimulus becomes "mono-oriented" when seen repeatedly in one orientation, and particular members of the stimulus class become difficult to discriminate in a non-normal orientation.

The separation of the children's Up-Inv curves after trial 9 (Fig. 1) is almost completely a function of the girls' superior Up performance (Fig. 2; in 10 of the 15 trials, mean per cent Up was greater for girls than for boys). Since this same finding has also been

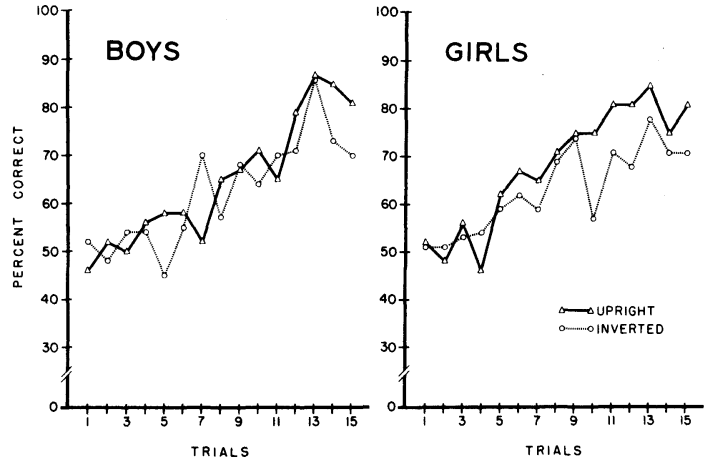

Fig. 2. Per cent correct responses of boys and girls to four paired associates. Each data point for the boys is derived from 12 Ss in the Upright curve and $14 \mathrm{Ss}$ in the Inverted curve; for the girls, $12 \mathrm{Ss}$ in the Upright and $17 \mathrm{Ss}$ in the Inverted.

obtained in two pilot studies, it deserves comment. One possible explanation is that girls are psychologically more mature than boys of equal chronological age. If this were the explanation, it suggests that the asymptote of the function under investigation - the effect of "overlearning" of upright faces-is approximately at the age of the Ss in this study. The reader's attention is directed to an earlier study of perceptual inversion (Brooks \& Goldstein, 1963) where recognition of inverted familiar faces was directly related to chronological age, a finding which may appear to conflict with the present data, viz., in former study, younger children "disturbed" by inversion; in latter study, younger children less "disturbed" than adults by inversion. The relationship of these two findings is being studied in further experiments.

\section{References}

Brooks, R. M., \& Goldstein, A. G. Recognition by children of inverted photographs of faces. Child Develpm., 1963, 34, 10331040 .

Goldstein, A. G, Learning and identification of mono-oriented visual stimuli as a function of their spatial orientation during learning and test trials. Paper read at Midwestern Psychological Association, Chicago, May, 1962. 